\title{
Video-rate or high-precision: A flexible range imaging camera
}

\author{
Adrian A. Dorrington ${ }^{* a}$, Michael J. Cree ${ }^{\mathrm{a}}$, Dale A. Carnegie ${ }^{\mathrm{b}}$, Andrew D Payne ${ }^{\mathrm{a}}$, \\ Richard M. Conroy ${ }^{\mathrm{a}}$, John P. Godbaz ${ }^{\mathrm{a}}$, and Adrian P. P. Jongenelen ${ }^{\mathrm{b}}$. \\ ${ }^{a}$ Department of Engineering, University of Waikato, Private Bag 3105, Hamilton, New Zealand. \\ ${ }^{\mathrm{b}}$ Department of Physical and Chemical Sciences, Victoria University of Wellington, PO Box 600, \\ Wellington, New Zealand.
}

\begin{abstract}
A range imaging camera produces an output similar to a digital photograph, but every pixel in the image contains distance information as well as intensity. This is useful for measuring the shape, size and location of objects in a scene, hence is well suited to certain machine vision applications.

Previously we demonstrated a heterodyne range imaging system operating in a relatively high resolution (512-by-512) pixels and high precision ( $0.4 \mathrm{~mm}$ best case) configuration, but with a slow measurement rate (one every $10 \mathrm{~s}$ ). Although this high precision range imaging is useful for some applications, the low acquisition speed is limiting in many situations. The system's frame rate and length of acquisition is fully configurable in software, which means the measurement rate can be increased by compromising precision and image resolution.

In this paper we demonstrate the flexibility of our range imaging system by showing examples of high precision ranging at slow acquisition speeds and video-rate ranging with reduced ranging precision and image resolution. We also show that the heterodyne approach and the use of more than four samples per beat cycle provides better linearity than the traditional homodyne quadrature detection approach. Finally, we comment on practical issues of frame rate and beat signal frequency selection.
\end{abstract}

Keywords: Range image, ranging, video rate, precision, linearity, resolution, three dimensional, alias

\section{INTRODUCTION}

A range imaging camera produces an output similar to a digital photograph, but every pixel in the image contains distance information as well as intensity. This is useful for measuring the shape, size and location of objects in a scene, hence is well suited to certain machine vision applications. For example, the use of range data instead of intensity data can simplify the segmentation of objects in an image, especially when the objects contain high contrast patterns or artifacts. Figure 1 shows an object imaged with the Waikato University Range Imager as both intensity (a) data and range data (b) with grey scale representing distance from the camera. The object can be segmented with significantly greater ease using the range data rather than the intensity data. A simple threshold operation can be performed to isolate the object of interest, lying within a specific distance band, from the background. Figure 1c and $1 \mathrm{~d}$ demonstrate this successful segmentation despite the wide variety of grayscale intensities both in the object and background.

*a.dorrington@waikato.ac.nz; phone+64-7-858-5062; fax +64-7-838-4835

Image Processing: Machine Vision Applications, edited by Kurt S. Niel, David Fofi,

Proc. of SPIE-IS\&T Electronic Imaging, SPIE Vol. 6813, 681307, @ 2008 SPIE-IS\&T · 0277-786X/08/\$18 
Prior to thresholding, the range data was pre-processed to remove invalid measurements. This pre-processing consisted of two simple steps. Firstly, range data was discarded when the returned intensity was too low (resulting in very noisy results). Secondly, the range data at the edge of objects was discarded. Invalid range data can occur at the edges of objects because the edges do not normally lie on pixel boundaries. At object edges it is common to have a mixture of range data from two (or more) objects in one pixel, resulting in a range value in-between the actual values. This also occurs where objects are not in sharp focus. Invalid range data is detected by assuming an object's surface does not contain rapid changes in range data. A pixel of range data is discarded if there is a change larger than five centimeters between the adjacent horizontal or vertical pixels.

In addition to simplified object segmentation, by its nature, a range imaging machine vision system can also provide information about an object's location and distance from the camera, simplifying any robotic interaction. In figure 1a it is difficult to tell if one particular object is smaller than the other or if it is more distant. However, this is abundantly clear in the range image (figure 1b).

a)

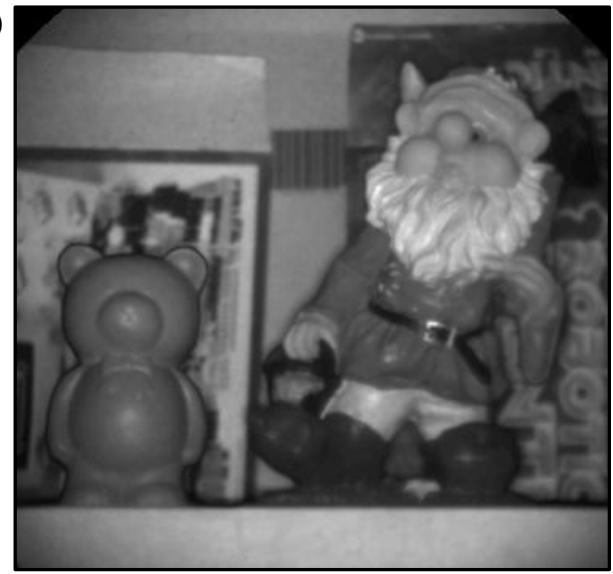

c)

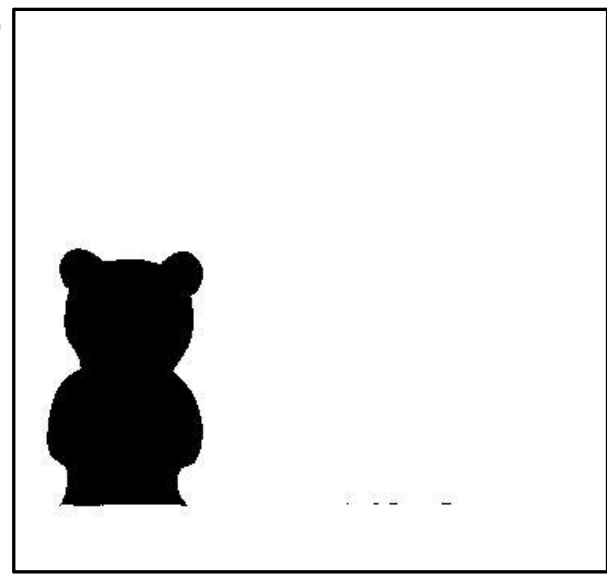

b)

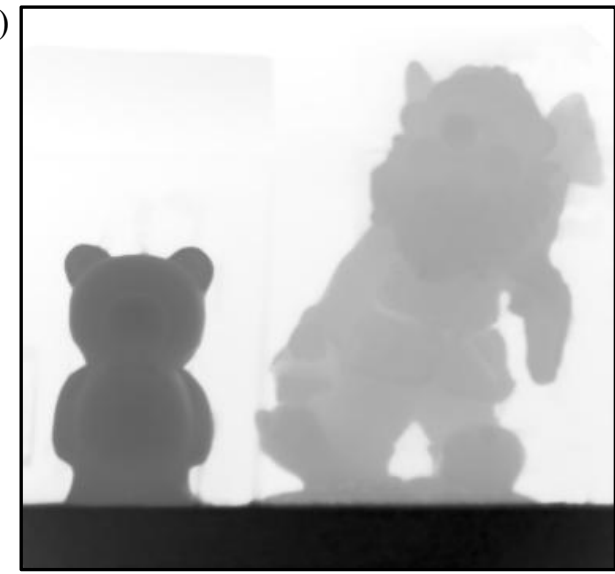

d)

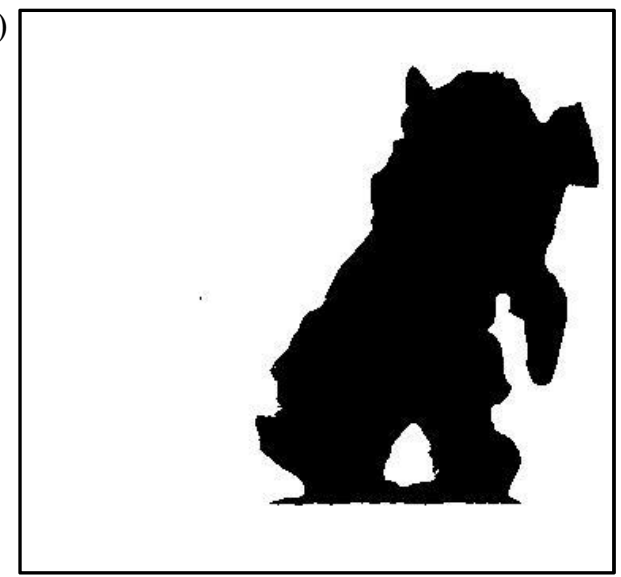

Figure 1 - Example of a scene containing high contrast objects imaged with the Waikato Range Imager. a) Intensity data from range imager. b) Range image represented as greyscale, where black is closest to the camera. c) Object close to the camera segmented by thresholding the range data. d) Object in the middle distance segmented by thresholding the range data.

Several full-field solid-state range imaging systems have been demonstrated with a wide variety of imaging quality and capabilities. A high-speed shuttering mechanism, or image modulation mechanism, operating in the region of $10 \mathrm{MHz}$ to 
$100 \mathrm{MHz}$ is a fundamental requirement for range imaging systems. Range imaging systems can be categorized as either internal shuttering or external shuttering by the image modulation technology employed.

Internal shuttering systems utilize a custom designed image sensor that integrates the shuttering or image modulation function into the image sensor itself ${ }^{1-6}$. Although this approach allows for small and efficient range imaging systems, they are currently limited in imaging resolution to around $25 \mathrm{k}$-pixels due to the nature of the image sensor. These systems also tend to exhibit range precision limited to sub-centimeter levels. Such limitations mean that this technology is not suitable for many machine vision applications.

External shuttering systems use a standard CCD or CMOS image sensor coupled to an external image modulation device $^{7-15}$. This external modulation device is typically an Image Intensifier simply because it is one of the few technologies that is capable of modulating an image at $100 \mathrm{MHz}$. Compared to the internal shuttering method, much higher imaging resolutions can be achieved with this approach, but the equipment is significantly more complicated and data acquisition is usually much slower.

Previously we have demonstrated an external shuttering range imaging system call the Waikato Range Imager (that was used to acquire the data for figure 1) operating in a high resolution and high precision configuration ${ }^{13-15}$. In this mode, range images of 512 by 512 pixel resolution and best case distance precision of $0.4 \mathrm{~mm}$ has been demonstrated at acquisition rates of one range image every ten seconds. Although this high precision range imaging is useful for some applications, the low acquisition speed is limiting in many situations. Fortunately, the Waikato Range Imager is very flexible in its mode of operation and can be operated at higher measurement rates by compromising resolution and range measurement quality.

In this paper we compare the ranging performance and trade-offs in terms of linearity, precision, and acquisition rate in different configurations; and comment on practical issues of frame rate and beat signal frequency selection. We also demonstrate the flexibility of our range imaging system by showing an example of video-rate ranging and high precision ranging digital zooming features, which are useful in a wide variety of different machine vision applications.

\section{HOMODYNE AND HETERODYNE RANGE IMAGING}

A number for range imaging techniques exist ${ }^{16}$, but the majority of solid-state full-field range imaging systems utilize the amplitude modulated method for indirect time-of-flight distance measurement. In this approach, a scene is illuminated with an intensity modulated light source, and imaged with a camera system that is also gain modulated or shuttered. The propagation delay from the light source to an object in the scene and back to the camera causes a phase delay in the illumination modulation envelope. The light received by the camera is effectively mixed (or multiplied) with the camera's shutter modulation.

In the popular homodyne configuration, the same modulation signals are applied to both the illumination and the camera, resulting in a DC or fixed gray level proportional to distance ${ }^{7}$. Light received from an object close to the camera experiences a small propagation delay, and hence a small phase shift in its modulation envelope, producing a relatively bright pixel or set of pixels. A more distant object causes a larger propagation delay, resulting in a larger phase difference and a less bright pixel.

The observed pixel brightness can be converted into measured distance values, but other effects such as background lighting and scattering properties of the object must also be considered. The normal approach is to use a quadrature detection system, requiring the acquisition of four measurement images with a relative phase differences between the two modulation signals incrementing in 90 degree steps ${ }^{2}$. 
An alternative to phase stepping is to employ the heterodyne approach, where there is a small frequency offset between the two modulation signals ${ }^{14}$. In the heterodyne case, the mixing process of the camera's shuttering modulation produces a beat signal. This beat signal has a frequency equal to the difference between the modulation frequencies, and also retains the relative phase difference. Consequently, distance can be determined by analysis of the phase value of the time history of a given pixel. A heterodyne system in which the camera frame rate is configured to be four times the beat signal frequency is analogous to the quadrature four-phase stepped homodyne approach.

The quadrature approach is popular because of its simplistic implementation and computation. This approach is most appropriate only where at least one of the modulation signals is truly sinusoidal, but in many practical implementations the modulation signals are square waves. Each set of harmonics in the modulation signals mix with each other at the camera producing harmonics in the beat signal, as illustrated in figure 2a. For the case of square wave modulation signals, a triangle shaped beat signal is produced. When only four samples are acquired for each beat cycle, as is the case for the quadrature detection approach, the Nyquist frequency is equal to twice the beat signal frequency.

Consequently, the odd valued beat signal harmonics are aliased onto the beat signal fundamental, causing contamination of the beat signal and interference in the phase determination. A simulation measuring phase from a triangle shaped beat signal using the quadrature approach is plotted in figure $2 \mathrm{~b}$. This figure shows a linearity plot of actual phase vs. measured phase and a phase error plot. Note the phase measurement accuracy errors exceed $\pm 1 \%$.

a)

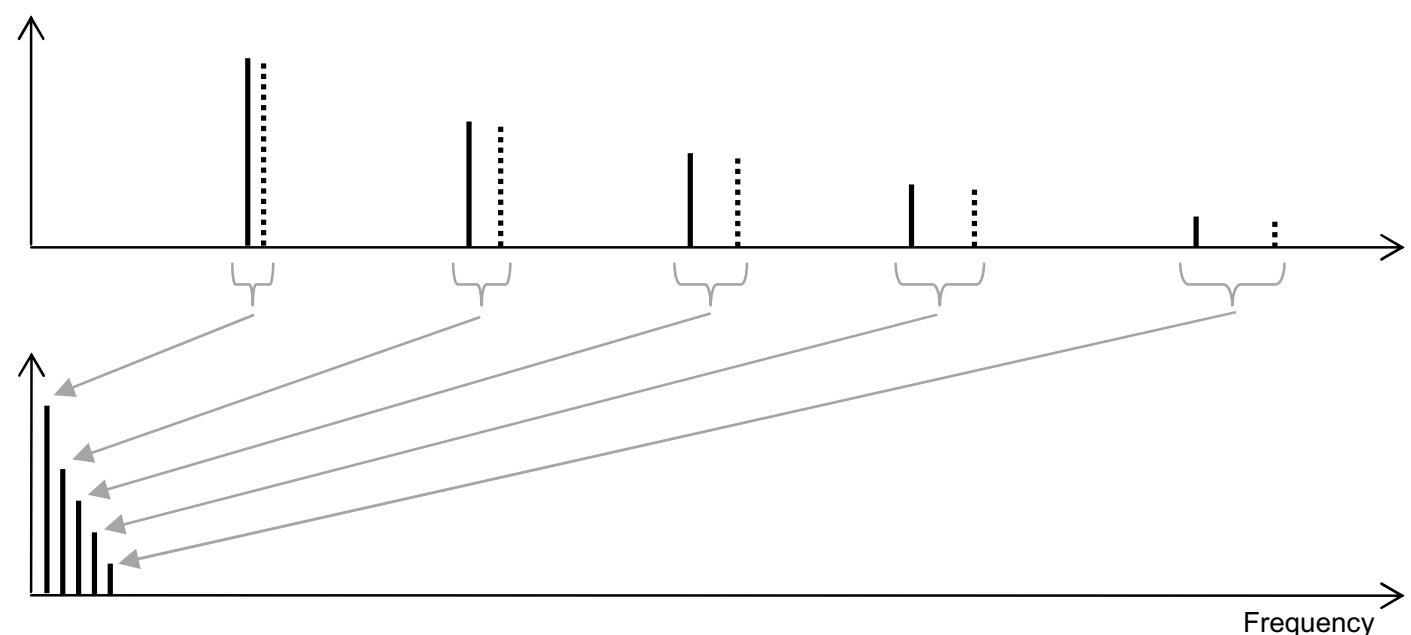

b)
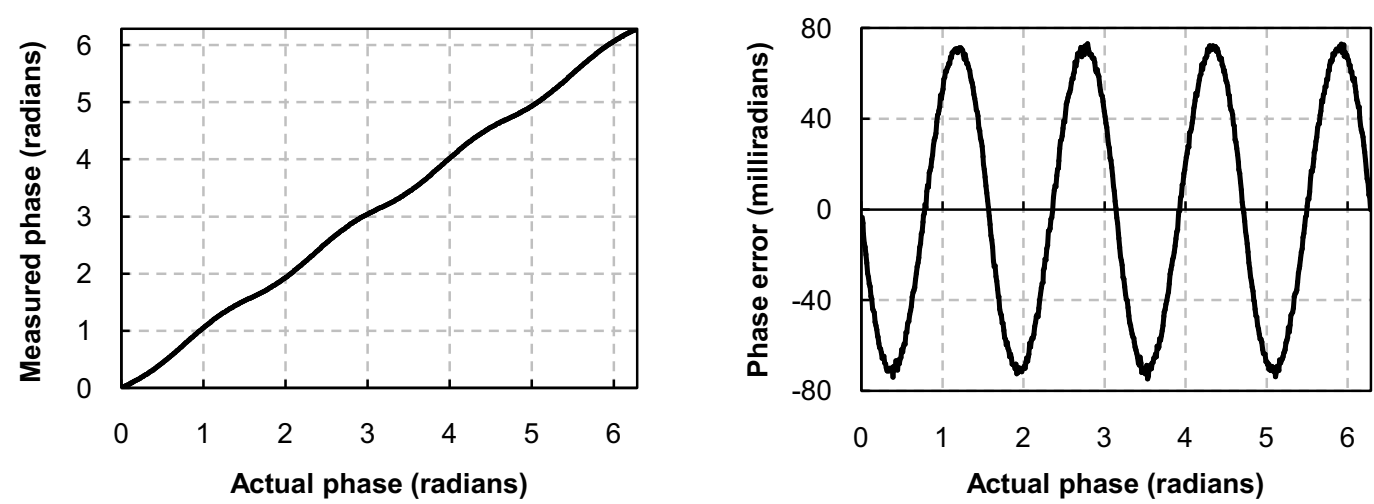

Figure 2 - The generation and effect of beat frequency harmonics. a) Frequency domain representation of two modulation signals (solid and broken lines) with a small frequency difference. b) Simulation of phase determination from a beat signals containing harmonics represented as a linearity plot (left) and an error plot (right). 
As shown in figure 3, the homodyne approach can be considered as sampling the beat signal with a delta function because the relative phase of the two modulation frequencies remains constant during the camera's integration period. Consequently, harmonics are aliased at their full amplitude. With the heterodyne approach, the frequency difference between the modulation signals effectively causes a continuous phase change during the integration period, corresponding to a rectangular sampling function

$$
\operatorname{rect}\left(\operatorname{tnr} f_{b}\right) \stackrel{F T}{\Leftrightarrow} \frac{1}{n r f_{b}} \operatorname{sinc}\left(\frac{\pi f}{n r f_{b}}\right)
$$

where $n$ is the number of samples per beat cycle, $r$ is the ratio of the sampling period to the integration time, and $f_{b}$ is the beat frequency. As the frequency of the harmonics increase, their amplitude decreases in accordance with the averaging effect of a finite sampling period. Therefore, the aliasing of harmonics has less impact in the heterodyne system because the harmonic amplitudes are reduced compared to the homodyne system.
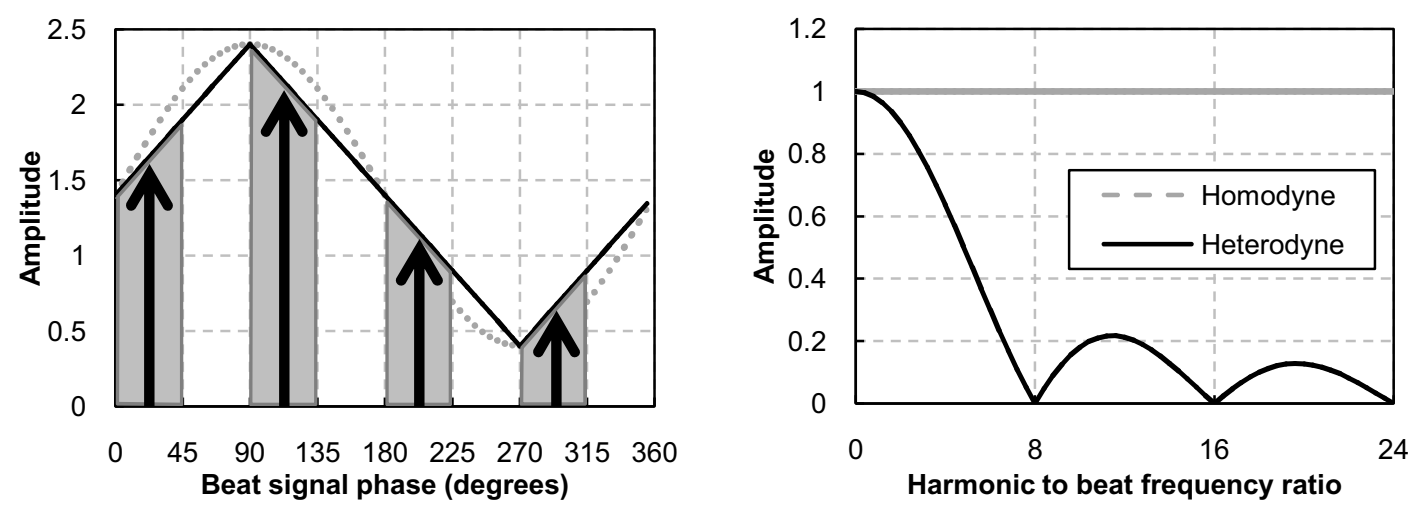

Figure 3 - Effective beat signal sampling (left) for the homodyne (dark arrows) and heterodyne (shaded areas) techniques for a triangle beat waveform, and the frequency response (right) of both methods. Assuming $r=2$ (in equation 1).

The problem of harmonic contamination can be further reduced by careful choice of the number of samples per beat cycle, thereby reducing the number of harmonics that can interfere with the measurement. Consider the example of square wave modulation signals and the homodyne approach with five phase steps of $72^{\circ}$ (instead of four steps of $90^{\circ}$ ), or the heterodyne approach with 5 samples per beat cycle. In principle, the ninth harmonic is the first harmonic to be aliased such that it interferes with the beat signal fundamental. The square wave modulation produces a triangle wave beat signal, so there are no even harmonics and the ninth harmonic is already attenuated $38 \mathrm{~dB}$ purely due to the inversesquare relationship of triangle wave harmonics. With the heterodyne method this ninth harmonic is attenuated by an additional $19 \mathrm{~dB}$ (from equation 1 assuming the integration time is equal to the sampling period), totaling $57 \mathrm{~dB}$ attenuation. For comparison, in the quadrature approach the third harmonic is the most dominant harmonic to interfere with the measurement, and it is only attenuated by $19 \mathrm{~dB}$ for the homodyne method or $29 \mathrm{~dB}$ for the heterodyne method. In practice, the beat signal will not be perfect triangular wave and will contain some even order harmonics. Therefore, in the above example, the fourth and sixth harmonics will also be aliased and interfere with the fundamental. However, their amplitudes should still be substantially lower than the third harmonic providing better results than the quadrature approach.

To highlight the effect of sample number selection on phase measurement linearity, simulation results are presented in figure 4. The simulation is of heterodyne beat signal processing over the range of four to 30 samples per beat cycle with a signal to noise ratio of $54 \mathrm{~dB}$. In each case, the root-mean-squared (RMS) linearity error was calculated from the difference between determined phase and actual set phase, over $2 \pi$ radians in 0.1 milliradian steps. The phase determination precision was also calculated as the standard deviation of 32 repeated simulations at each phase step, 
averaged over all phase steps. Note the significant improvement in RMS linearity errors for odd numbers of samples per beat cycle. At nine samples per beat cycle, the linearity error falls below the measurement precision (errors due to random variations).

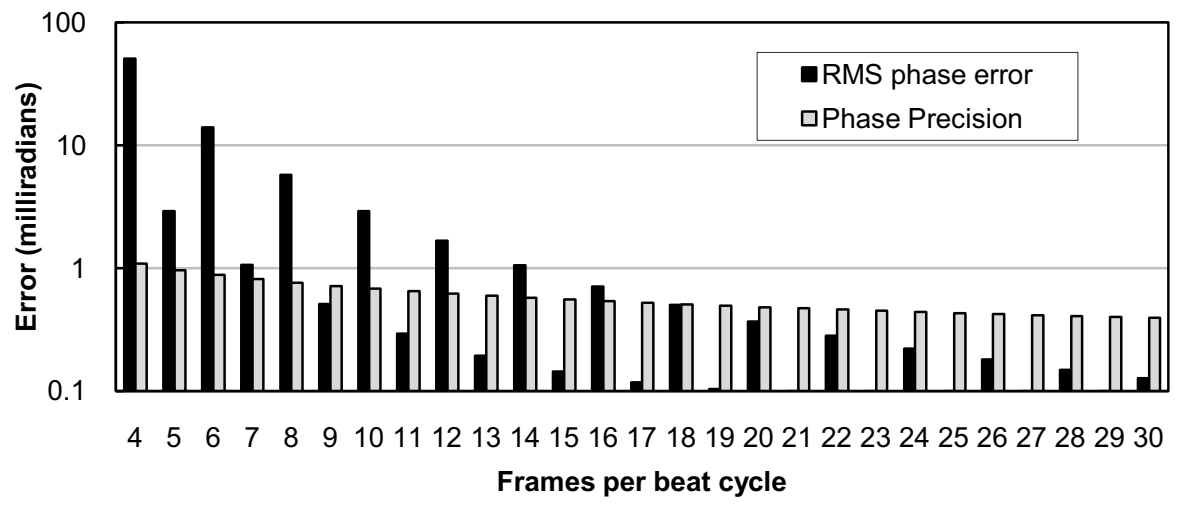

Figure 4-Simulated linearity and precision errors for four to 30 frames per beat cycle.

\section{THE WAIKATO RANGE IMAGER}

The Range Imaging Group in the Department of Engineering at the University of Waikato (New Zealand) has developed a full-field solid-state range imaging system based on a heterodyne imaging apparatus ${ }^{13-14}$, shown in figure 5 . This system illuminates a scene with a combination of four $80 \mathrm{~mW}$ laser diodes that can be intensity modulated up to $100 \mathrm{MHz}$. The scene is imaged via a standard Nikon F-mount camera lens attached to a MCP125 image intensifier (Photek Ltd, East Sussex, UK), lens coupled to a Pantera 1M60 digital video camera (DALSA Corporation, Waterloo, Ontario, Canada) digital video camera. Image gain modulation is achieved by applying a modulation signal to the image intensifier's photocathode.

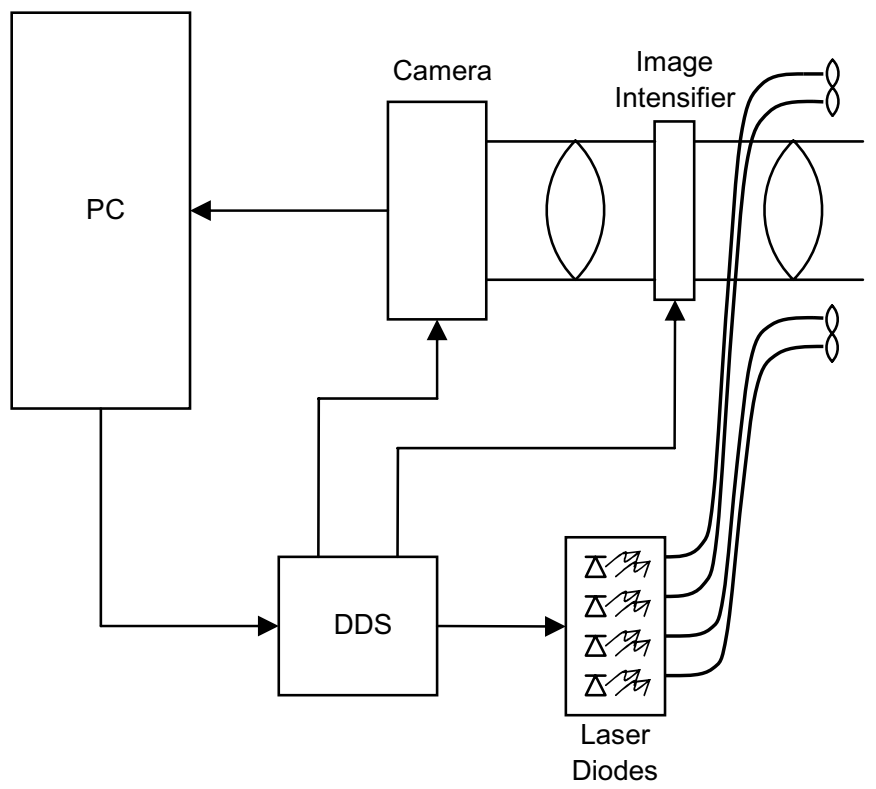

Figure 5 - Waikato Range Imager set-up 
We typically use a manual focus 80 to $200 \mathrm{~mm}$ zoom lens, but the system's field-of-view can easily be adjusted by changing the imaging lens. The laser illumination is delivered to the imaging head by $1 \mathrm{~mm}$ diameter fiber optic-cables (one for each laser diode) butt-coupled to the laser diodes, and are arranged symmetrically about the imaging axis to provide co-axial illumination. The illumination divergence is controlled by a $6 \mathrm{~mm}$ diameter lens at the output of each fiber-optic cable, and can be adjusted to approximately match the field of view of the imaging lens.

Modulation frequencies between $10 \mathrm{MHz}$ and $90 \mathrm{MHz}$ with frequency differences (between the illumination and photocathode modulation) between $0.1 \mathrm{~Hz}$ and $60 \mathrm{~Hz}$ are commonly used. A group of AD9952 (Analog Devices, Norwood, MA, USA) direct digital synthesizer (DDS) circuits are used to generate the modulation signals with appropriate frequency stability ${ }^{15}$. A camera frame trigger signal is also generated by the DDS to allow frequency locking between the beat signal and the sampling signal. This frequency locking is used to ensure an exact integer number of samples are acquired in a beat cycle, which allows simple inner-product (single bin DFT) phase determination processing. A microprocessor provides the control functions for the signal generators, image intensifier gain, and laser level, and an RS-232 serial link to a desktop computer. The computer also contains a frame grabber to acquire the images from the digital camera, and the overall control and data processing software.

Previously, high precision range results were obtained by ensuring a high signal-to-noise ratio (SNR) with low frame rates and long imaging integration times, as well as acquiring a large number of video frames to process. However, for situations requiring high range acquisition speeds, the video frame rate must be increased and the number of frames processed decreased, resulting in a reduction in precision. The Waikato Ranger's video acquisition is fully configurable in software, so the frame rate and number of frames captured can be easily adjusted. This means that it can be reconfigured to operate in either a high precision or a video-rate range acquisition mode, and can switch between modes rapidly. Increasing the video frame rate necessitates reducing the integration time and therefore compromising the signal to noise ratio. The pixel read-out speed of the camera imposes an upper limit to the frame rate. However, the pixel binning feature can be utilized to achieve even higher frame rates by reducing the image resolution.

\section{EXPERIMENTAL SET-UP}

As with the simulation described above, a measure of linearity was obtained by finding the RMS of the repeatable phase determination errors, and the precision due to random errors was measured by finding the standard deviation of multiple measurements. As described in more detail below, the results differ markedly for different modulation frequencies. To highlight the differences, measurements were taken at modulation frequencies of $12 \mathrm{MHz}$ and $80 \mathrm{MHz}$, close to the low and high modulation frequency limits of the system.

The system configuration is summarized in table 1. In order to achieve high-speed real-time range measurements suitable for machine vision applications, the camera binning was set to 8-by-8 pixels. This allowed the frame rate to be increased to maximum at the expense of image resolution. Also in an effort to maintain the maximum possible range measurement rate, all range acquisitions use only one beat cycle per measurement. To find the linearity, a phase sweep was performed with the number of samples per beat cycle varying between three and thirteen. The beat frequency and frame rate were adjusted for each sweep to obtain the best combination of frequencies. Care was taken to ensure the camera frame rate stayed as close as possible to 205 frames per second and there were an exact integer number of samples per beat cycle.

Not all combinations were possible because of the resolution limitation of our signal generator. DDS signal generators are limited to output frequencies in quantized steps defined by their internal clock rate divided by the maximum value of the tuning word. In our case, the frequency resolution is approximately $0.0931 \mathrm{~Hz}$. It is often easier to approximate the frequency resolution as roughly $0.1 \mathrm{~Hz}$ (or use the integer frequency tuning values native to the DDS), and convert to actual operating frequencies as required. Note that the values presented in table 1 are actual signal frequencies. 
Table 1 - Waikato ranger configuration for linearity and precision measurement

\begin{tabular}{ll}
\hline Characteristic & Value \\
\hline Binning & 8 -by- 8 \\
Imaging resolution & $128-$ by-128 \\
Modulation frequency & $80 \mathrm{MHz} / 12 \mathrm{MHz}$ \\
Camera frame rate & $204.5 \mathrm{~Hz}-205.4 \mathrm{~Hz}$ \\
Frames per beat cycle & $3-13$ \\
Beat frequency & $68.5 \mathrm{~Hz}-15.7 \mathrm{~Hz}$ \\
Beat cycles acquired & 1 \\
Range measurement rate & $68.5 \mathrm{~Hz}-15.7 \mathrm{~Hz}$ \\
Lens focal length & $80 \mathrm{~mm}$ \\
Lens aperture & $4.5 \mathrm{f}$-number \\
\hline
\end{tabular}

Given a specific number of frames per beat cycle, only certain candidate combinations of camera frame rate and beat signal are possible. For example, using the $0.1 \mathrm{~Hz}$ resolution approximation, running the video camera at 220 frames per second and requiring 7 frames per beat cycle would need a beat frequency of $31.428 \mathrm{~Hz}$. This is not possible as the beat frequency is not a multiple of the frequency resolution. The closest functional operating frequencies are a beat frequency of $31.4 \mathrm{~Hz}$ and a frame rate of $219.8 \mathrm{~Hz}$.

The phase determination errors were found by performing a phase sweep similar to that described in the simulation above, and subtracting the known phase from the measured phase. Each sweep consisted of a range measurement at two degree phase steps from 0 to 358 degrees. To help reduce noise, phase measurements were averaged across a sub-region of 65-by-65 pixels near the center of the range image, and across eight repeated measurements. The phase steps were generated electronically by controlling the relative phase offset of the two modulation signals through the DDS. A planar diffuse white surface was used at the target object, and throughout the measurement process the physical set-up was not moved. To avoid the influence of the linearity errors, the precision was calculated individually for each phase step in the sweep as the standard deviation of 41 image pixels near the image centre and across the eight measurement repetitions, and averaged for all phase steps.

\section{RESULTS}

The worst case phase measurement errors are shown in figure 6 . These are at four and three frames per beat cycle at $12 \mathrm{MHz}$ and $80 \mathrm{MHz}$ modulation respectively. Figure 7 plots both the phase linearity errors and the phase determination precision for all acquired measurements. Note that the phase errors for $80 \mathrm{MHz}$ modulation at three frames per beat cycle are disproportionately large compared to the rest of the results, but over-all, the higher modulation frequency exhibits a lower level of linearity errors and better precision. When converting these phase errors to distance errors, the values for the higher modulation frequency decrease even further because the phase to distance conversion factor is proportional to the wavelength of the modulation signal ${ }^{13}$.

These measured results differ significantly from the simulated data shown in figure 4 because the modulation signals are not perfectly square, hence the beat waveform is not a perfect triangle wave. The actual beat waveforms shown in figure 8 are generated by acquiring a video sequence of the system running at 220 frames per second with a $1 \mathrm{~Hz}$ beat frequency. To reduce noise in the plot a group of 1024 pixel near the centre of the image have been averaged. The beat signal for $12 \mathrm{MHz}$ modulation rate is approximately of a triangular wave, but is truncated because the duty cycle of the modulation signals is not exactly $50 \%$. At $80 \mathrm{MHz}$, bandwidth limiting of the drive electronics reduces the number of harmonics in the modulation signals, resulting in a beat signal that has a more sinusoidal shape. 


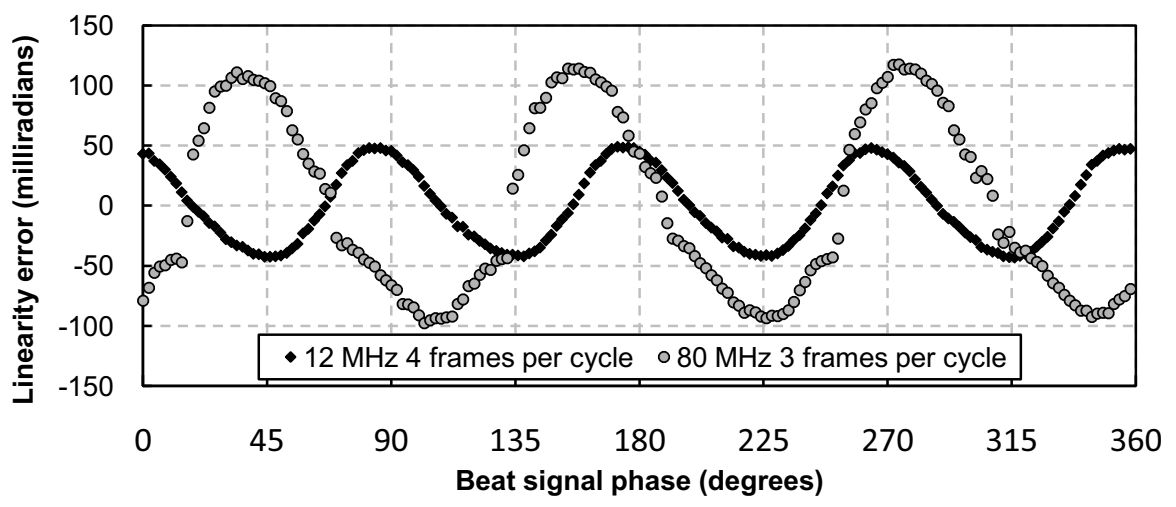

Figure 6 - Linearity errors for four frames per beat cycle at $12 \mathrm{MHz}$ modulation and three frames per peat cycle at $80 \mathrm{MHz}$ modulation.
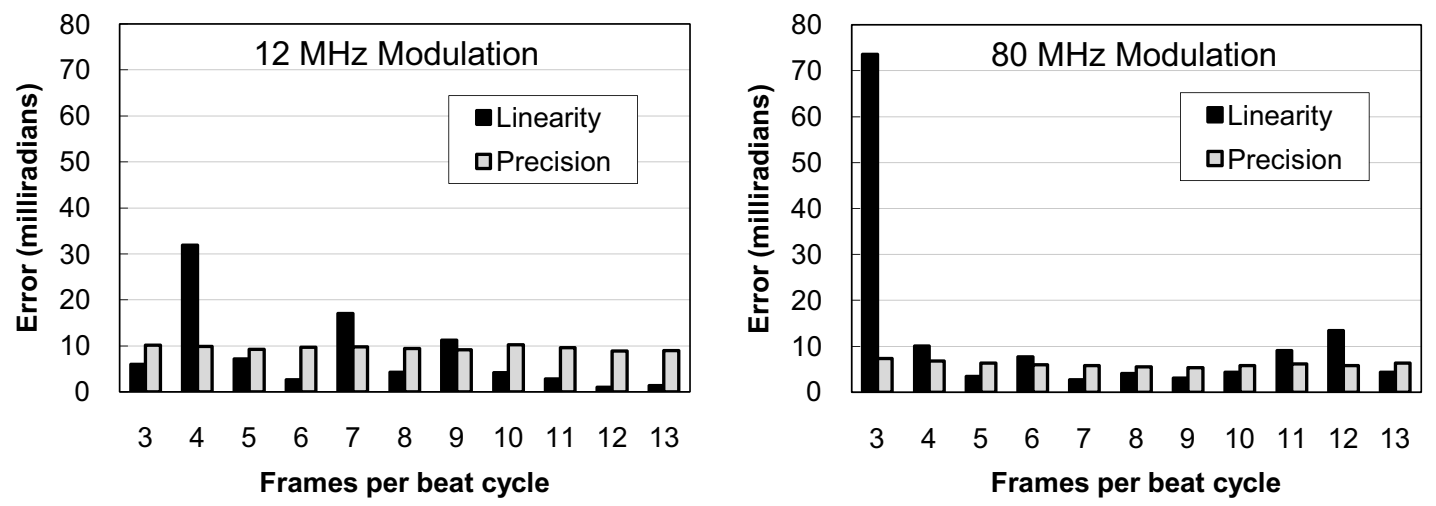

Figure 7 - Linearity phase errors and phase determination precision for $12 \mathrm{MHz}$ and $80 \mathrm{MHz}$ modulation with frames per beat frequency ranging from three to thirteen.
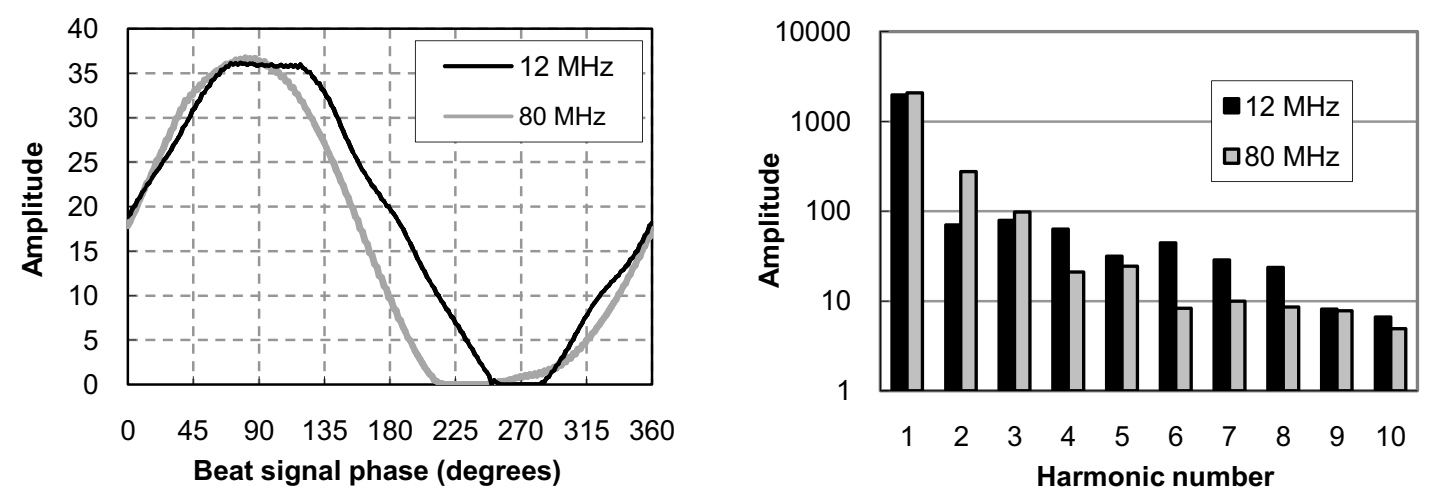

Figure 8 - Beat signal plots for $12 \mathrm{MHz}$ and $80 \mathrm{MHz}$ (right) modulation frequencies in the time domain (left) and the frequency domain for the first 10 harmonic (right). 
Also shown in figure 8 in the Fourier transform of the beat signals showing the first 10 harmonics. The harmonic levels are generally lower for the $80 \mathrm{MHz}$ modulation, consistent with the band limiting of the modulation electronics. The notable exception is the second harmonic, which is approximately 4 times larger for $80 \mathrm{MHz}$ modulation. Because the second harmonic is aliased onto the fundamental at 3 frames per beat cycle, the large second harmonic is a likely cause of the significantly larger linearity errors for $80 \mathrm{MHz}$ modulation at 3 frames per beat cycle.

A good compromise between measurement rate and ranging performance is five frames per beat cycle at $80 \mathrm{MHz}$ modulation rate. At this setting the RMS linearity errors are below the measurement precision (as can be seen in figure 7), and the system is capable of acquiring around 44 range measurements per second. The measurement precision could be improved by decreasing the measurement rate (to 15 measurements per second, for example), thereby allowing a slower frame rate, longer integration time, and better signal to noise ratio. Alternatively, the imaging resolution could be increased to 256-by-256 pixels, and the range measurement rate decreased to 30 per second (due to a maximum camera frame rate of 156 frames per second). The resolution could be increased even further to 512-by-512 with 20 measurements per second. These resolution and measurement rate combinations are camera dependent, and would change if different camera hardware (with different native resolutions and maximum frame rates) was used.

Figure 9a shows the same scene as figure 1, but measured at 128-by-128 pixels imaging resolution with a $33 \mathrm{~ms}$ measurement time, corresponding to real-time range imaging at 30 measurements per second. The digital zoom feature is illustrated in figure $9 \mathrm{~b}$, where the region-of-interest in the scene is measured in high-resolution mode with a ten second high-precision measurement time. In this configuration, the full frame image is set to 512-by-512 pixels, but the region of interest is set to 128-by-128 pixels to match the image size of the high-speed measurement in figure $9 \mathrm{a}$. The zoomed image shows significantly less noise, as a result of the longer measurement time.

a)

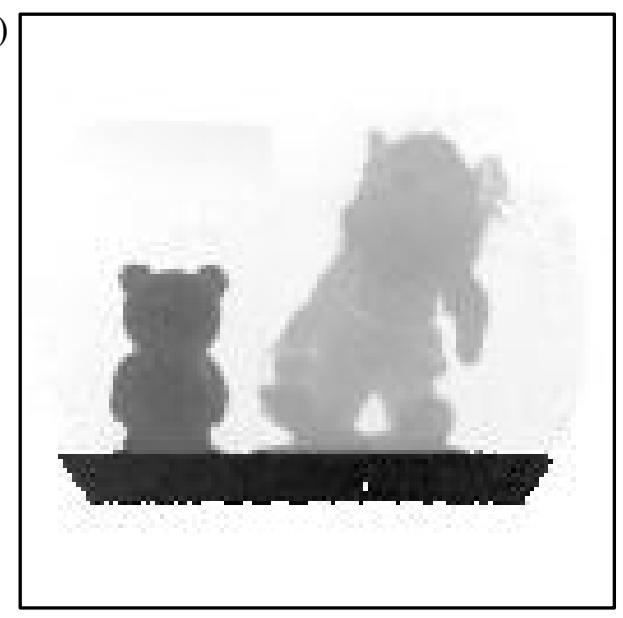

b)

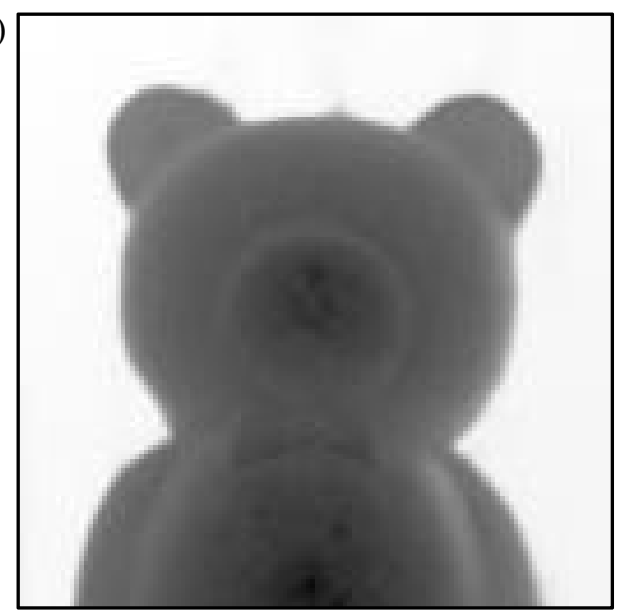

Figure 9 - Demonstration of digital zoom in range images. a) Real-time low-precision range measurement of the full imaging field at 128-by-128 pixel resolution. b) 128-by-128 pixel region-of-interest of a high-precision high-resolution range image.

Our current digital video camera hardware does not support a true region of interest function, so the digital zoom image shown in figure 8 above is a simple crop of the full-field high-resolution capture. Consequently, there is no speed advantage in implementing the digital zoom. However, an alternative camera with full region-of-interest support could easily be substituted if a specific application warranted it. 


\section{CONCLUSION}

Although the use of homodyne quadrature phase detection with square wave modulation is common in full-field solidstate range imaging systems, such a configuration is non-optimal. This paper has shown that both the heterodyne approach and the use of more than four samples per beat cycle provide better linearity (individually and in combination). With particular reference to the Waikato Range Imager, we have also shown that it is possible to trade-off operating speed vs. imaging resolution and ranging precision. This provides the ability to quickly and easily configure the system for the optimal performance suiting a particular application or situation, and to change this configuration in real-time. The example of video-rate low-precision range imaging of a full scene, and high-precision low-speed digital zoom imaging of a small region of the scene is presented.

\section{REFERENCES}

1. P. Gulden, D. Becker, and M. Vossiek, "Novel optical distance sensor based on MSM technology," IEEE Sensors Journal, 4(5), 612-618 (2004).

2. R. Lange, P. Seitz, A. Biber, and S. Lauxtermann, "Demodulation Pixels in CCD and CMOS Technologies for Timeof-Flight Ranging," Proc. SPIE, 3965, 177-189 (2000).

3. R. Miyagawa and T. Kanade, "CCD-based range-finding sensor," IEEE Transactions on Electron Devices, 44(10), 1648-1652 (1997).

4. T. Oggier, M. Lehmann, R. Kaufmann, M. Schweizer, M. Richter, P. Metzler, G. Lang, F. Lustenberger, and N. Blanc, "An all-solid-state optical range camera for 3D real-time imaging with sub-centimeter depth resolution (SwissRanger)," Proc. SPIE - Optical Design and Engineering, 5249, 534-545 (2004).

5. T. Spirig, M. Marley, and P. Seitz, "The multitap lock-in CCD with offset subtraction," IEEE Transactions on Electron Devices, 44, 1643-1647 (1997).

6. D. Stoppa, L. Viarani, A. Simoni, L. Gonzo, and M. Malfatti, "A new architecture for TOF-based range-finding sensor," Proc. IEEE Sensors, 1, 481-484 (2004).

7. J.P. Anthes, P. Garcia, J.T. Pierce, and P.V. Dressendorfer, "Non-scanned LADAR imaging and applications," Proc. SPIE - Applied Laser Radar Technology, 1936, 11-30 (1993).

8. J. Busck and H. Heiselberg, "Gated viewing and high -accuracy three-dimensional laser radar," Applied Optics, 43, 4705-4710 (2004).

9. S. Christie, S. L. Hill, B. Bury, J. O. Gray, and K. M. Booth, "Design and Development of a Multi-Detecting 2Dimensional Ranging Sensor," Measurement Science \& Technology, 6, 1301-1308 (1995).

10. M. Kawakita, K. Izuka, R. Iwama, K. Takizawa, H. Kikuchi, and F. Sato, "Gain-modulated Axi-Vision camera (high speed high-accuracy depth-mapping camera),” Optics Express, 12( 22), 5336-5344 (2004).

11. T.C. Monson, J.W. Grantham, and S.W. Childress, "Characterization of scannerless LADAR," Proc. SPIE - Laser Radar Technology, 3707, 409-419 (1999).

12. B. Stann, M. Giza, D. Robinson, W. Ruff, S. Sarama, D. Simon, Z. Sztankay, “A scannerless imaging ladar using a laser diode illuminator and FM/cw radar principles," Proc. SPIE- Laser Radar Technology and Applications IV, 3707, 421-448 (1999).

13. A. Dorrington, A. Payne, R. Conroy, M. Cree, D. Carnegie, "Achieving sub-millimeter precision with a solid-state full-field heterodyning range imaging camera," Measurement Science and Technology, 18, 2809-2816 (2007). 
14. D. A. Carnegie, M. J. Cree, A. A. Dorrington, A High-Resolution Full-Field Imaging System, Review of Scientific Instruments, 76, CID 083702 (2005).

15. A. D. Payne, D. A. Carnegie, A. A. Dorrington, and M. J. Cree, "Full Field Image Ranger Hardware", The third IEEE International Workshop on Electronic Design, Test \& Applications, Kuala Lumpur, Malaysia,17-19 January, 263-268 (2006).

16. P.J. Besl, “Active, optical range image sensors”, Machine Vision and Applications, 1, 127-152 (1988). 